\title{
Massive Open Online Courses (MOOCs): A Comparative Analysis of the Main Platforms
}

\author{
Lidia Mihaela FUNIERU, Florin LĂZĂROIU \\ Bucharest University of Economic Studies, Romania \\ funierulidia11@stud.ase.ro, lazaroiuflorin11@stud.ase.ro
}

Massive open online courses have been recently introduced as a modern learning method mainly due to the great progress made in the fields of Information Technology and, moreover, the ever increasing number of technologies supported by the Internet. The rapidly growing diversity of MOOC providers and the popularity of this fast emerging learning option influenced this analysis to compare the main characteristics of the most popular MOOC platforms at this time, Coursera and edX. The platforms have been compared from both a technical and a user perspective and each detailed feature was graded based on its strengths and weaknesses.

Keywords: Characteristics, Comparison, Open Online Course Platform, Technical, User

1 Introduction

A massive open online course (MOOC) represents a means to learning in an online environment which provides open access for unlimited participants, enabling more than traditional learning models and materials including recorded lectures, quizzes, interactive forums and communities [1]. The necessity for MOOCs came along with the digital era, the need of gathering knowledge at a fast pace and lifelong learning, therefore new approaches had to be considered to fulfil the learner's needs [2].

The purpose of this analysis is to identify the main characteristics of this type of learning by comparing the most used open access learning platforms and the facilities they offer considering that they generate interest from different parties, not only universities but also business capitalists who can identify an opportunity worth exploring in this field. This is one of the main reasons why start-ups like Coursera have begun collaborating with wellknown universities around the globe, others have decided to make their courses available online through a platform like edX and even large companies are considering to use a MOOC approach in the sector of higher education [3]. MOOCs promise to offer an alternative to higher education which breaks the pattern of known university-level education models, therefore it is important to explore the engine behind open online course platforms.

Because massive open online courses have been recently introduced as a modern learning method, the field of research in MOOCs is in its early stages and the available literature analyzing MOOC platforms is relatively limited. The reviewed literature aims to uncover the features which determine the quality of an online course platform compared to another.

From a general perspective MOOCs have a series of specific features that should be part of any platform that belongs to this area of education. In [4] five principles were recommended for designing a MOOC. The first one refers to the meaningful content provided by the platform in spite of its massive feature. This is achieved by avoiding large flows of ideas by keeping the topics on point, by using stimulating materials or examples shared by more experienced students on the discussion boards. The second principle is the engagement of the students who enroll, which represents a challenge also because of the size of the online courses which makes it impossible for instructors to have individual contact with all the students and influence their will to finish what they started. This can be accomplished with the help of traditional teaching techniques or with social engagement that can be provided through networked interaction forms such as forums or 
chat rooms. The measurement of the student's progress and the course's effectiveness represents the third principle. The online characteristic of MOOCs enables a correct evaluation of the course's efficacy. This principle is useful for both the student who can see his own improvement and the instructors who can improve their materials when these prove ineffective. The accessibility is yet another fundamental aspect of MOOC design since the education level of the students who enroll may vary from none (in matters of higher education) to $\mathrm{PhD}$ and the same goes for the extent to which they are familiar with the topic they want to tackle. Finally, the last principle is the scalability which refers to the ability to grow rapidly in size without making significant changes to the existing structure.

When analyzing comparative studies about massive online platforms, more practical characteristics are discovered. One of the key aspects focused on by the research community is the learning theory applied. According to [5] the commonly used MOOCs of our time (Udacity, Coursera, edX, Udemy) fall into the xMOOC category which tends to adopt a traditional behaviorist model. They are regarded as the most widespread and popular MOOCs and for this reason they have attracted the most attention from the researchers. In the case of xMOOCs, the learner is provided with video presentations covering the course curriculum, but learners have the freedom to adapt the pace of studying to match their own learning speed, while, at the same time, being restricted to complete the whole course within a given number of weeks [6].

From [7] where aspects of the main existing platforms (Coursera, edX, Udacity, FutureLearn) were analyzed from a general perspective as well as from a user perspective and a technological one, other MOOCs characteristics are identified which can help classify the earlier mentioned platforms: financial aim of the provider (profit or nonprofit), the accreditation or availability of verified certificates and the number of students enrolled. The study manages to conclude that the purpose of the different platforms may vary, some focusing mainly on developing their technology as edX does, while others such as Coursera have a strategy that consists in the rapid expansion of their partnerships (universities and other types of research and education institutions). A similar comparative analysis was conducted in [8] which compared platforms Coursera and edX along with a third platform, NPTEL, also from user and technical perspective. The study was focused on dimensions such as use of multimedia, certificate courses and availability of mobile applications, language support and use of social media. All three of the platforms were graded on each perspective with Coursera scoring the highest grade on most analyzed dimensions while the Indian provider received very low grades. Furthermore, the author offered suggestions and emphasized the importance of some of the analyzed aspects. Other key aspects which should be considered in any MOOC comparison are presented in [9]. The approach of this study was more hands-on in the fact that accounts and courses were created on edX and Moodle MOOC, in order to experience their characteristics. Course structures, communication tools, tests and grading were considered to determine the similarities between the compared platforms and in the same time decide which their original approaches are.

Most of the reviewed characteristics represent the core of our comparative analysis of MOOCs and will be further detailed in regard to specific platforms, Coursera and edX.

\section{The Research Method}

This study employed a research method based on the own experience of the authors with the purpose of exploring the characteristics of MOOC platforms from user and technical perspectives in order to understand the capabilities they require to motivate online learning. To reach this goal, platforms Coursera and edX were accessed and courses from the computer science study domain were analysed to properly identify their strengths and weaknesses. Coursera and edX were 
chosen as a case study because of their popularity among online education platforms. Both emphasize on collaborating with high ranking institutions known for being intensive in doing research such as Stanford and Princeton in the case of Coursera and Massachusetts Institute of Technology (MIT), Harvard and Berkeley for edX. What makes them different at first sight are the size of their course catalogue and the number of collaborations, Coursera being the leader. The online course providers also differ from a financial perspective, Coursera being a profit organization and edX not. This study analyzes their main features (illustrated in Figure 1) in an attempt to determine the most relevant.
In order to evaluate the considered characteristics a qualitative scale (Low/Medium/High) is used. Because Coursera and edX are the most wide spread MOOC platforms [7] and none of their features is poorly implemented, the "Low" score was not attributed to any characteristic, but was kept for uniformity. In order to assign a "Medium" ranking, the analyzed characteristic has to be satisfactory or can be improved upon in regard to technology or user experience. The "High" rating is awarded when the platform applies the highest technology standards available for the investigated aspect or when original approaches are introduced.

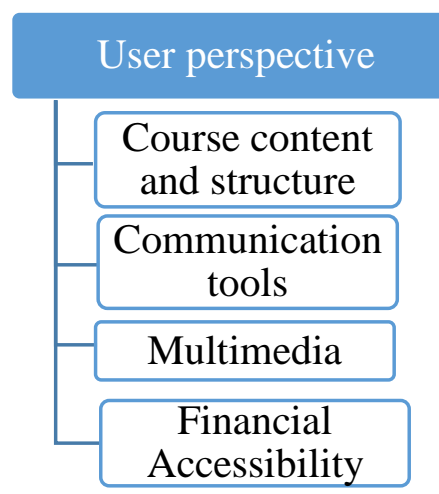

Technical perspective

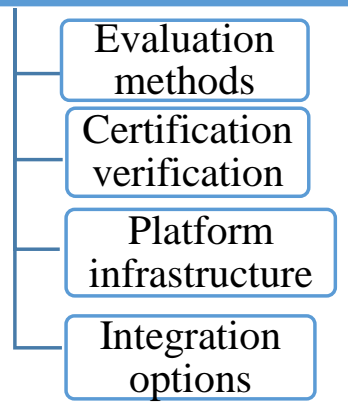

Fig. 1. Considered characteristics

\section{The Research Results}

This section focuses on presenting the most relevant characteristics identified by our analysis as results of the research.

\subsection{Course structure and content}

The course content variety and quality are a vital part of a learning platform since they represent the core of offering high-level education material. Most courses from edX are from science domains such as mathematics, physics or computer science, while Coursera has a wider catalogue, classes from a greater number of domains being at reach. Even though Coursera offers significantly more courses, edX seems to make up for quantity with quality. Also, as far as computer science content goes, edX provides a more dynamic learning method in the online environment. Moreover, Coursera seems to have less rigorous classes in comparison to edX which is known for trying to apply on-campus standards in the online learning environment and provide the user with a feeling of following college-level courses. Yet, the lack of rigor is not necessarily a downside of Coursera's content since it allows more students to take part, eliminating the prerequisites.

Although MOOC courses do not share a common pattern and do not present a general structure, both platforms offer four to twelve weeks long classes with content divided into variable length sections. Most of these sections include activities which can be completed within a one week period. On Coursera a weekly class consists of a one to three hours long video lecture with periodic in-lecture quizzes. EdX's video lectures are 
similar in length, but are divided in smaller segments and alternate with questions to make sure that the student understands the information. The schedule of this classes varies by class, some may be timed, some like most edX courses usually have a strict schedule, but some offer the student the advantage of being self-paced, meaning that he can go through classes whenever he has the time. Coursera even offers courses ondemand.
As for user experience, although the sections of different classes from Coursera may be utterly distinct they share the same layout so that courseware, announcements, deadlines, new content and forum posts can be easily identified. Both platforms group related course materials by week, but Coursera is better organized, all key sections being rapidly identified as can be seen in Figure 2. Figure 3 shows edX's less intuitive layout

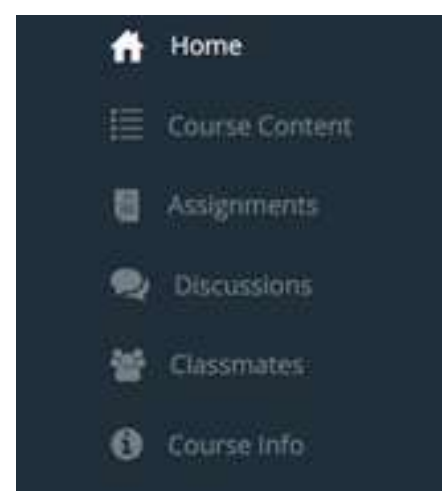

Fig. 2. Coursera's content organization

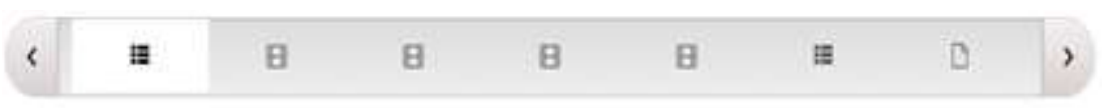

Fig. 3. EdX's content organization

\subsection{Communication tools}

Communication is important for students in order to reach a greater level of engagement with the course and the two platforms have similar approaches regarding it. Coursera makes use of web forums to facilitate communication between students and mentors. Each course has its own forums, separated into general discussion ones and others dedicated to each course module. In the case of edX, while most courses feature web forums with discussions separated by topic, some edX courses allow students to connect with classmates through official communities created on popular social networks. Despite edX occasionally offers this option, Coursera has the upper hand due to having more structured and intuitive discussion means.

While the two platforms offer student-student communication tools, in both cases interaction with the teacher is minimal or completely absent. This may be due to the teacher's great workload and overwhelming class sizes, making contact with individual students difficult. Also, neither Coursera nor edX have integrated a type of live chat feature which could offer more fluid conversations between classmates. For this reason, most students feel the need to resort to other exterior communication tools that offer instant messaging.

\subsection{Evaluation methods}

The importance of assessment methods is reflected in the user's ability to track his performance and have a correct evaluation of his work. Depending on the topic of each course, a range of different grading and assessing techniques are applied in both Coursera and edX. The learning platforms use diverse types of online tests, such as practice sessions, midterm exams, final exams, 
practical assignments, final projects and video embedded quizzes. For multiple choice questions, which can be taken separately or as part of the video lectures, Coursera uses automatic grading. Auto-grading is also used to evaluate programming assignments (for MOOCs covering Computer Science topics). Another grading method adopted by Coursera is calibrated peer review (CPR) assessment, which can be used for open response assignments, such as essays. CPR requires that every time students submit an assignment, they are required to review and give feedback to a few other students, based on a defined rubric set by the course teacher. This type of grading is very useful when important assignments cannot be graded by a computer and students can gain valuable learning experience while reviewing someone else's work. EdX uses almost the same assessment techniques as Coursera for grading open response assignments: peer grading, self-grading, machine grading, staff grading. Each EdX course has different types and numbers of assignments that have to be carried out throughout the course before attempting to pass the final exam.

What sets edX apart is its new automated essay scoring (AES). According to [10], the system trains itself to grade essays using machine learning algorithms (ML) based on how a human grader manually evaluates the first hundred essays. AES systems build statistical models by observing certain patterns and characteristics of human grading. For example, correlations can be made between the human assigned score and the length of the essay, the frequency of grammar errors or the vocabulary used. AES can also use complex computational elements from the field of Natural Language Processing (NLP), such as automatic summarization, discourse analysis or sentiment analysis. The advantage of AES is that it can provide students with instant qualitative and quantitative feedback on their work.
Also, while Coursera offers only means of tracking your assignments feedback, edX offers a Progress section which displays the grades received for different quizzes, assignments and exams in a detailed fashion. Overall, edX seems to give a greater importance to the assessment process than Coursera, channelling more energy into developing new methods of giving feedback to students.

\subsection{Use of multimedia}

Nowadays, MOOCs should rely less on text as a manner of delivering knowledge to students because of the easily available video technology and other forms of interactive content that can be embedded into courses. The use of multimedia and interactive learning are an effective and engaging manner of delivering knowledge. Aside from plain text and images, both Coursera and edX allow partner universities to create video-based course content and most MOOCs available on these platforms stream video content. The possibility to download the video or the transcript, add subtitles or play the video in High Definition is present on both platforms, but edX also makes it possible to choose the quality of the downloaded video file, the language of the transcript, to download PDF presentation slides and students can also choose to only download the audio file.

EdX holds another advantage regarding multimedia in the fact that some courses feature lab sections with interactive embedded applications. An example is the course UC3Mx: IT.1.1x Introduction to Programming with Java which embeds an external resource called Codeboard to allow students to write and test their code in the online environment (Figure 4). Another example are the labs featured in course MITx: 6.002.1x Circuits and Electronics 1, which allow students to build circuits in an embedded tool (Figure 5). Unlike these, Coursera's programming assignments must be solved on the student's personal computer. 


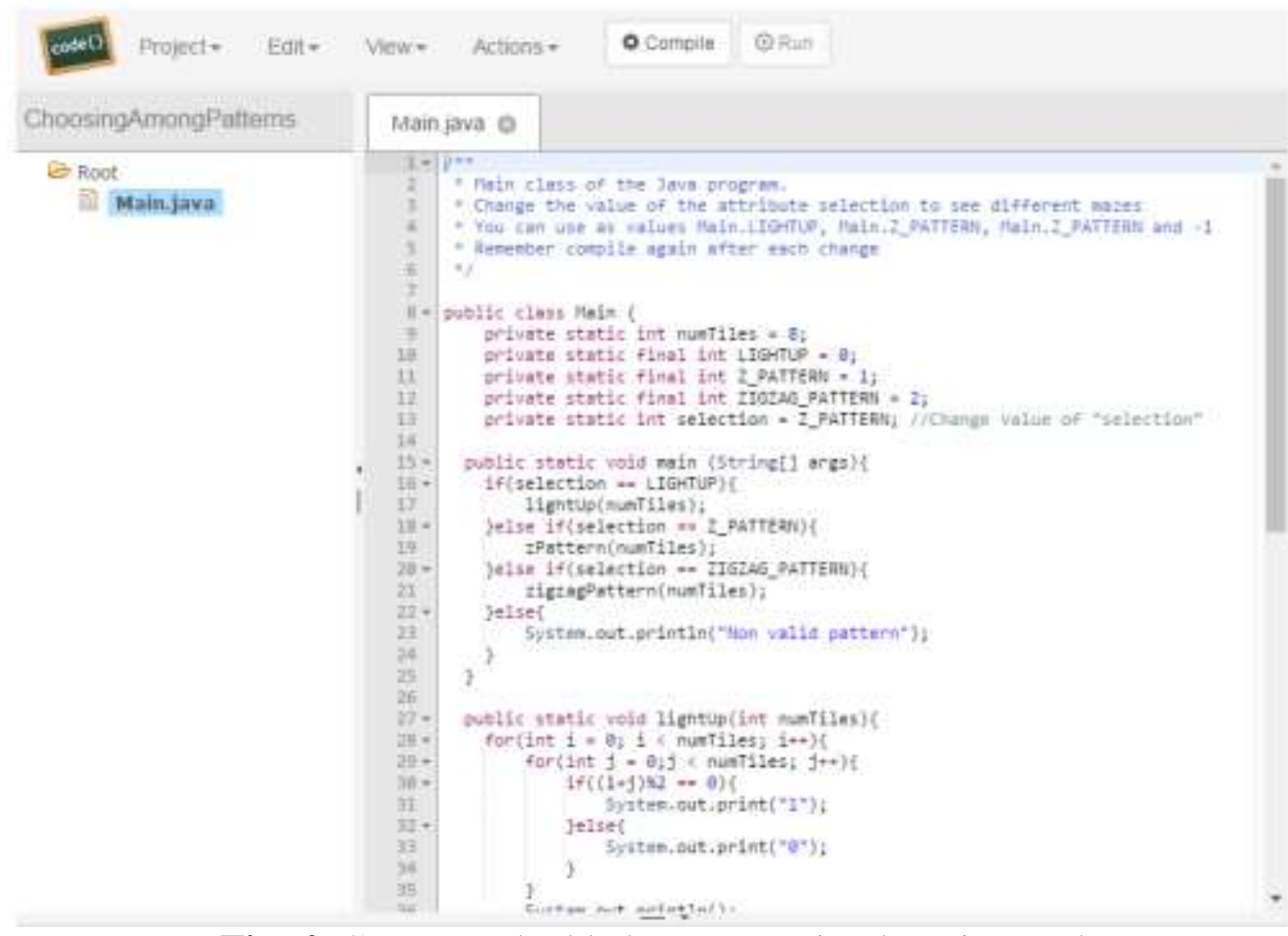

Fig. 4. Course embedded programming learning tool

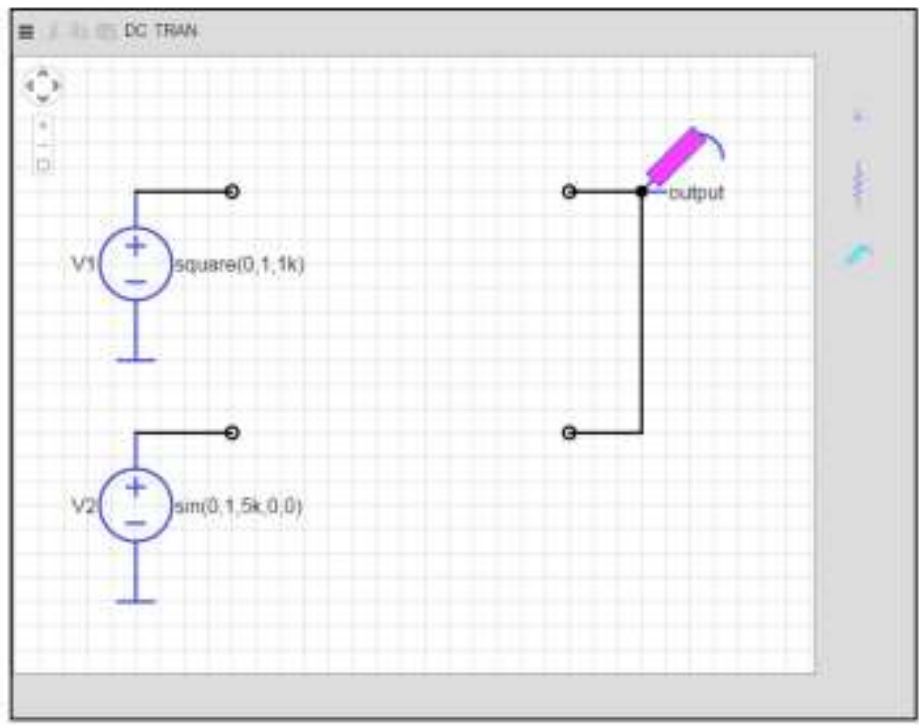

Fig. 5. Course embedded circuits learning tool

\subsection{Certificates and verification}

Certificates are a way for students to build their professional qualifications which proves valuable for them and their careers. Each edX and Coursera finalized class can provide the student with proof of enrollment or in exchange for a fee with a verified certification. In the case of edX the verification is made through facial recognition using a real time webcam photograph of the student and one of a government issued identification. Coursera's verification is more complex because it adds another approach consisting in a signature track. Based on the student's typing style, a keystroke signature is created. Behind the keystroke dynamic are the rhythm and manner in which an individual types using a keyboard. After the measurement of the time for a key to be pressed and the time between two consecutive presses, the data is processed by a neural algorithm which determines a biometric 
template of the user's typing pattern used for future authentication.

Completed courses on both platforms may provide the student with university credit, but if an institution chooses to award that credit does not mean that another is bound to accept it. In the computer science field edX has more credit eligible courses than Coursera.

\subsection{Financial accessibility}

Similar to any other business, MOOC platforms need to generate enough revenue in order to sustain themselves and invest in the evolution of the platform. Coursera has a profit oriented business model and generates revenue from three main sources: fee-based courses which charge students a fee to gain access to graded assignments, specializations, which feature a series of four to six courses ending with a capstone project that requires students to put the acquired knowledge to use in order to earn a certificate and, finally, Course Certificates which give students the opportunity to earn a verified certificate by paying a flat fee. In spite of all these fees, Coursera still offers many free courses and also features a Financial Aid Program that can help students with financial difficulties. On the other hand, edX is one of the few nonprofit MOOC providers, but still requires revenues in order to be self-sustainable and achieve its declared mission of offering "access to high-quality education for everyone, everywhere" [11]. This mission is reflected in the fact that students enrolling in edX courses have the option to audit the course free of charge, but without the advantage of earning a verified certificate. Similarly to Coursera, edX also offers the option of applying for financial aid, but even if the aid application is successful, the student is offered a partial discount. Although the initial concept of edX was free online learning, high-quality content on digital platforms comes at a cost, so the concept is now shifting to meet the increasing demand of certificate courses, training in skill-specific domains and corporate learning.

\subsection{IT Infrastructure}

While the two platforms cannot be compared based on this criteria, the software technology is relevant when creating a web-based platform to support higher education. The information for this characteristic was extracted from sources [12] and [13].

The edX web application was created using the Python programming language and the application framework Django also written with Python. Coursera was programmed with Scala and its corresponding Scala written open source framework Play. In opposition to Python which supports multiple programming paradigms such as procedural, object-oriented and functional, Coursera's server-side code was written in a programming language that has full support for functional programming. In terms of client-side programming, edX was created using JavaScript and the Backbone.js framework. Sass, an extension language for CSS is being used for styling the front-end of the application, alongside the Bourbon library which is a framework designed for it. The browsing-side of Coursera is written in a similar fashion using JavaScript, but with a different framework, Express.js. For the web server the two course platforms benefit from the same technology, Nginx (which is the actual web server) on the cloud platform Amazon Web Services.

The student information from edX is stored in MySQL relational databases while the courses are kept in a document-oriented database, MongoDB, falling into the NoSQL category because relational database rules do not apply to it. The available videos are served either from Youtube or from the web service designed for online file storage Amazon S3. In the beginning Coursera also used MySQL for data storage, but in time it migrated to Apache Cassandra, another NoSQL database. This platform also makes use of the Amazon S3 web service provided by Amazon Web Services.

Both platforms have created the mobile correspondent of their application, these being available for the iOS and Android operating systems. EdX's application allows the user to stream or download videos to keep up with the 
weekly class work. Coursera's mobile version also enables the two options of downloading to watch later or streaming a video, quiztaking, course browsing or even enrolling.

\subsection{Integration options}

Some MOOC platforms may feature methods to allow external developers to create web, desktop and mobile applications that have the ability to interact with the platform, either to retrieve certain course related data or to send information to the platform, for example, with the purpose to update a user account or enroll a user in a course.

Being an open-source platform, edX comes with a web-based platform named Open edX which is the main software behind edX and other learning websites. The complex architecture of Open edX has been made public as it was conceived with the purpose of creating and analyzing MOOCs.

Open edX offers integration facilities in the form of Platform APIs that support the JSON (JavaScript Object Notation) data format and are based on the design principles of representational state transfer (REST). Some of the currently available APIs are the Enrollment API 1.0 and the User API V 1.0. The first one allows retrieving user enrollment data and enrolling a user in a course while the latter is used to update user account and preferences information. Furthermore, the Learning Management System of Open edX can act as a consumer of Learning Tools
Interoperability (LTI), so external LTI tools can be integrated in the course. Another notable API is the AI Grading API which allows interaction with edX's automated grading system. Also, custom JavaScript applications can be integrated in the courses using edX Studio, which is a course development environment. EdX offers a detailed documentation regarding all of these integration options.

The other analyzed platform, Coursera, offers a set of APIs on its beta version App Platform which developers can use to integrate their own applications with the platform. These APIs are less reliable than those offered by edX and they even come with a warning from Coursera: "things may break, change or go away without warning" [12]. The Coursera integrations currently available are OAuth2 APIs, full LTI 1.1 compliance (used to integrate external learning tools), course catalog APIs and Shibboleth integration. OAuth 2.0 is an authentication protocol which enables users to delegate an application to gain access to an HTTP service on behalf of the user. In this category, the available APIs are oriented on manipulating enrollment and basic profile information. The catalog APIs offer access to information regarding courses, sessions and trainers.

Table 1 summarizes the results of our comparison by grading each characteristic with a value from the defined scale.

Table 1. Comparison Results

\begin{tabular}{|l|l|l|l|l|}
\hline $\begin{array}{l}\text { User } \\
\text { perspective }\end{array}$ & $\begin{array}{l}\text { Structure \& } \\
\text { Content }\end{array}$ & Communication Tools & $\begin{array}{l}\text { Multimedia } \\
\text { Resources }\end{array}$ & $\begin{array}{l}\text { Financial } \\
\text { Accessibility }\end{array}$ \\
\hline Coursera & High & Medium & Medium & Medium \\
\hline edX & High & Medium & High & High \\
\hline $\begin{array}{l}\text { Technical } \\
\text { perspective }\end{array}$ & $\begin{array}{l}\text { Certification } \\
\text { Verification }\end{array}$ & Evaluation methods & Infrastructure & Integration \\
\hline Coursera & High & Medium & High & Medium \\
\hline edX & Medium & High & High & High \\
\hline
\end{tabular}

Coursera has the advantage over edX in a content related perspective because it covers multiple areas of education, not only science. It also provides a more detailed overview of course content and related materials, making the user's interaction with the platform more intuitive and the browsing experience more rapid. But edX balances the situation with the 
rigorous structure of its classes and the quality of the materials.

Generally, both platforms are rich in multimedia features, but edX has invested most in the technology used to deliver the course content with the featured interactive labs that come with some of its computer programming courses. EdX is also superior in terms of evaluation techniques because of the automatic grading system for grading essays. Overall it seems to also give a greater importance to the assessment process than Coursera, by developing new methods of giving feedback to students and tracking their performance.

Both Coursera and edX offer decent communication tools, but neither have implemented a form of instant interaction and there is no direct communication between students and course instructors, therefore they could do more to meet the user's needs. Another similarity is the option to obtain proof of participation in a class, a verified certificate or earn institutional credit from eligible courses, but Coursera raises the bar with its verification method that includes a signature track for user authentication.

The two online education providers are created with modern, up-to-date software development technologies and seem optimization oriented. And although Coursera is taking small steps towards embracing the concept of open-source software by sharing some projects on Github, a web-based project hosting service, edX is notably more accessible for developers. This has an effect on the amount of technical information available on the internet about edX for interested developers or researchers which is more consistent and at reach. EdX is also more integration oriented due to the same opensource characteristic.

The results show that the platforms are similar and try to keep up with each other, but the analysed characteristics are convincing enough to state that edX has more potential.

\section{Discussions}

The findings presented in this paper are consistent with those of the existing literature in terms of orientation of MOOC platforms towards technology. Coursera is the dominating player on the MOOC market most likely because of its early start, diverse course domains and numerous higher education partners. Despite this, we concluded that edX has quickly caught up, driven by its focus on getting the most out of IT in order to offer students an engaging experience, as shown also by [7]. The quality of the materials on edX may be justified by the fact that the provider seems less interested than Coursera in making partnerships, but more focused on maintaining the level of commitment in regard to the materials they provide. This has been proven by its superiority (in comparison to Coursera) in matters of assessment methods, use of multimedia and integration possibilities.

Although previous studies, such as [8], have established that both platforms make use of multimedia at high standards, we have found that edX is slightly superior because it offers more options for the video content and gives greater importance to interactive learning tools. The results of this paper come to strengthen the ideas presented by [8] with regard to edX's higher openness of technology compared to Coursera and also those expressed by [9] regarding the mediocre communication tools offered by edX.

\section{Conclusions}

This study offers a new layer of knowledge about the characteristics of most widely used MOOC providers as an example for emerging platforms. Understanding these aspects and mostly what sets the platforms apart, either original approaches or intelligent technologies, is important for both users that can find the motivation to use a certain platform to accomplish their learning objectives and developers who can contribute to designing a platform that can help the students reach them. As MOOCs continue to grow, further research should focus on proposing improved features that could strengthen student engagement and attract more attention to this accessible way of gaining knowledge. 


\section{References}

[1] R. Baker, B. Evans, E. Greenberg and T. Dee, "Understanding Persistence in MOOCs (Massive Open Online Courses): Descriptive \& Experimental", in Proc. The European MOOCs Stakeholder Summit, Lausanne, Switzerland, 2014, pp. 5-10.

[2] L. Yuan and S. Powell. MOOCs and Open Education: Implications for Higher Education (A White Paper). Internet: http://publications.cetis.org.uk/wpcontent/uploads/2013/03/ MOOCs-andOpen-Education.pdf, 2013 [Mar. 14, 2016].

[3] C. Bonk, M. Lee, T. Reeves and T. Reynolds, MOOCs and Open Education around the World. New York: Routledge Publishing, 2015, preface.

[4] J. R. Drake, M. O'Hara and E. Seeman, "Five Principles for MOOC Design: With a Case Study", Journal of Information Technology Education: Innovations in Practice, vol. 14, pp. 125-143, 2015.

[5] M. Kesim and H. Altinpulluk, "A Theoretical Analysis of MOOCS Types from a Perspective of Learning Theories", in Proc. The 5th World Conference on Learning, Teaching and Educational Leadership, vol. 186, Prague, Czech Republic, 2014, pp. 15-19.

[6] M. Mangelsdorf. Massive Open Online Courses (MOOCs): An Assessment Based on Personal Experience. Internet: http://www.iedp.com/Pages/DocumentMa nager/Massive $\% 20$

Open\%20Online\%20Courses\%20_\%20F ull\%20Article.pdf, 2012 [Mar. 16, 2016].
[7] M. Sriram, "Comparative Analysis of Massive Open Online Course (MOOC) Platforms, in: Proc. The Fourth International Conference on Global Business, Economics, Finance and Social Sciences, Kolkata, India, 2015, ISBN: 978-1-63415-898-5

[8] P. Agrawal, A. Kumar and A. Agrawal, "Massive Open Online Courses: EdX.org, Coursera.com and NPTEL, A Comparative Study Based on Usage Statistics and Features with Special Reference to India", in CALIBER 2015: Shimla,HP. India: INFLIBNET Centre, 2015, ISBN: 978-93-81232-05-7, pp. 390402.

[9] M. Blagojević and D. Milošević, "Massive open online courses: EdX vs Moodle MOOC", in Proc. 5th International Conference on Information Society and Technology, Kopaonik, Serbia, 2015, pp.346- 351 .

[10] S. P. Balfour (2013, May). Assessing Writing in MOOCs: Automated Essay Scoring and Calibrated Peer Review ${ }^{\mathrm{TM}}$. Research \& Practice in Assessment Journal [Online]. 8(1). Available: http://rpajournal.com/dev/wpcontent/uploads/2013/05/SF4.pdf

[11] edX. About Us. Internet: https://www.edx.org/about-us [Apr. 6, 2016]

[12] Building Coursera. Internet: https://building.coursera.org/appplatform/ [Apr. 13, 2016]

[13] Open edX. Internet: https://open.edx.org/ [Apr. 13, 2016].

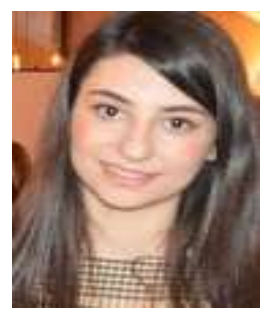

Lidia Mihaela FUNIERU has graduated the Bucharest University of Economic Studies, Faculty of Cybernetics, Statistics and Informatics in 2011 earning a Bachelor Degree in Economic Informatics. Currently she is attending the Research in Economic Informatics master program of the same faculty. 


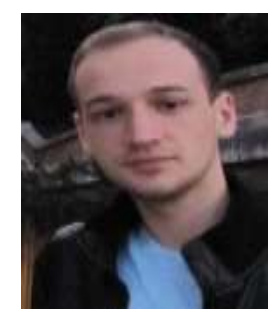

Florin LĂZĂROIU has graduated the Bucharest University of Economic Studies, Faculty of Cybernetics, Statistics and Informatics in 2011 with a Bachelor Degree in Economic Informatics. He is presently a student of the Faculty of Cybernetics, Statistics and Informatics, Research in Economic Informatics master program. 